\title{
Sfe: waiting for the big one
}

\author{
Juan José Curto*, Josep Castell, and Ferran Del Moral \\ Observatori de l'Ebre, (OE) CSIC - Universitat Ramon Llull, Roquetes, Spain \\ *Corresponding author: jjcurto@obsebre.es
}

Received 26 October 2015 / Accepted 19 April 2016

\begin{abstract}
Accurate measurements of the radiation delivered during the two largest solar flares ever observed are unavailable. In the case of the Carrington event (1858) the X-ray and UV radiation was not recorded, while in the case of the big flare which happened after the storm of 29-31 October 2003 we will call from now on as Halloween event (2003) the radiation saturated the X-ray radiometer. Despite many studies, a consensus regarding the real values of these events at the moment of maximum radiation has never been reached.

In this paper, we used an alternative approach to try and determine these values. We estimated the values from the perturbations they produced in the Earth's magnetism - these are known as Solar Flare Effects (Sfe). Firstly, we established an empirical relationship between the variation in the radiation (cause) and its effect on the magnetism (consequence). Then, using the inverse function, we estimated the energy flux of both events. We found that both flares can actually be classified as being larger than X45. Finally, we also calculated the return period for a Carrington-like flare. Assuming that this event had an intensity of about X45 according to our calculations - we estimated the return period to be $90 \pm 60$ years.
\end{abstract}

Key words. Sfe - Solar flares - Carrington event - Return period - Statistics

\section{Introduction}

Solar flare effects (Sfe) are rapid magnetic variations which are related to the enhancement of the amount of radiation produced during Solar flare events (Curto et al. 1994a). X-ray and EUV emissions are the main electromagnetic radiation which cause variations on the electronic density in the ionospheric layers. From the $\mathrm{F}$ to the $\mathrm{D}$ regions, there are electron density enhancements during solar flares and on Earth the magnetic signature of a flare is visible in the illuminated hemisphere.

Interest in the occurrence and frequency of solar flares has increased in the field of Space Weather because of the perturbations they produce on these variables - the electron density in the ionosphere or the earth's magnetic field. Both of these are used either actively or passively by key technological systems such as the GPS positioning/guidance system, HF communications, satellite communications, etc. (Lanzerotti 1979, 1983). The impact of severe Space Weather events on domestic and international networks can lead to huge economic costs (Cannon 2013; Schulte in den Bäumen et al. 2014). In this paper, we will concentrate on the radiative aspect of the flares producing Sfe and not on the ejected particles producing geomagnetic storms.

Although Carrington and Halloween events were at the top of the lists of outstanding solar flare effects identified in the scientific literature (Cliver \& Svalgaard 2004), there is no consensus regarding the real value of the two largest flares ever observed at their maximum points. The Carrington event (1858) was not registered, and the Halloween event (2003) saturated the X-ray detectors (Thomson et al. 2005). In fact, over the last few days of October 2003 a sequence of events happened, but the biggest one as regards radiation was that of 4 November, and, in this paper, it will be known as the Halloween event. This lack of consensus has led to different speculations regarding the true size of these events and we have decided to carry out new estimations from a different viewpoint.
The first observed solar flare, on 1 September 1859 (Carrington 1860; Hodgson 1860), is actually associated with the largest space weather event ever recorded (Cliver 2006). As reported by Cliver \& Svalgaard (2004), the Carrington event, as it is commonly called, is at the top of size-ordered lists of solar flare effects (Sfe), or magnetic crochet amplitudes as these observations used to be called.

Increasing interest in extreme Space Weather events for both practical and theoretical reasons has led to the re-examination of various aspects of the Carrington event, especially the flare size (Kiplinger \& Garcia 2004; Tranquille et al. 2009; Clarke et al. 2010; Cliver \& Dietrich 2013). As most of the mentioned studies agree, the Carrington flare may have been a large one, about the size of a X45-X50 solar flare.

The second flare event we study, that of 4 November 2003, is generally considered to be the most intense SXR event recorded during the satellite period, and occurred during the Halloween event sequence of flares (Gopalswamy et al. 2005). The GOES 1-8 $\AA$ emission in this event saturated at an SXR classification of X17.4. NOAA extrapolated the curve of the register and obtained a maximum of X28 (NOAA 2003). However, according to some authors it may have been a Carrington-like flare. Thus, Kiplinger \& Garcia (2004) reconstructed the light curve to estimate a peak classification of X30.6. Thomson et al. $(2004,2005)$ and Brodrick et al. (2005) estimated an SXR classification of X45 \pm 5 and X34-X48, respectively, but years later Tranquille et al. (2009) deduced a flare classification of X24.8 \pm 12.6 .

In this study we will assess the magnitude of these two flares by taking into account the magnetic signatures of the Sfe they created.

First, we shall look for an empirical relationship that can express the Sfe amplitude as a function of the flare energy. Then, using the inverse function, we can estimate the energy 
flux of the flares from the magnetic measurements of the Sfe, when the flare energy could not be measured.

Finally, we will calculate the return period of a Carringtonlike flare assuming that this event had an intensity of about X45 according to our calculations.

\section{Data set}

First, we identified a series of relevant events with simultaneously available radiation data from satellites and magnetic variations from magnetic observatories on Earth. Original data of X-ray flares from GOES satellites belonging to the National Oceanic and Atmospheric Administration (NOAA) have been used (ftp://ftp.ngdc.noaa.gov/STP/spaceweather/solar-data/ solarfeatures/solar-flares/x-rays/goes/). The database contains information of flares from 1 September 1975 to the present. In our case, the last date used is 21 May 2015.

As regards UV data, they belong to the CELIAS/SEM experiment on the Solar Heliospheric Observatory (SOHO) spacecraft and they were downloaded from the Space Sciences Center in the University of Southern California which hosts the data (http://www.usc.edu/dept/space_science/semdatafolder/ long/15_sec_avg/).

Finally, magnetic data were downloaded from the INTERMAGNET database (http://www.intermagnet.org/data-donnee/ download-eng.php) except for the Carrington case where we digitized the magnetogram of Kew Observatory (UK) for that day using our measuring technique (Curto et al. 1996).

For our data, we chose a sample rate of one minute.

\section{Sizing the biggest flares: what}

During a solar flare a huge amount of energy is liberated. When this reaches the higher levels of the earth's atmosphere it produces a large variation in the electron density for a short period of time. These sudden changes in electrical conductivity produce an increase in currents which, in turn, induce magnetic variations on Earth. Their sudden appearance makes them easily identifiable.

\subsection{Use of a mathematical model}

Our initial objective is to find a simple empirical relationship which relates the X-ray and UV-ray energy flux from a solar flare, with the value of the perturbation produced on the earth's magnetic field.

There are several issues involved which mean that determining the basic magnitudes of the problem is not easy. One of these is that, on a morphological level, the magnetic variation shape depends heavily on the spectral balance of the group of radiations making up the flare (Curto et al. 2016). The main flux (X-and UV-ray) vary over time and are different from one event to another, thus meaning it is difficult to make a prediction when we do not have direct measurements.

Another problem is that, regarding both radiation but especially magnetic variation, the signal of the event we aim to analyse (during the flare time) represents an increase in the value of a factor which is itself already variable in time and which depends on various, not always quantifiable, factors such as the neutral winds or the electric conductivity in the dynamo region which modulates electric currents producing the magnetic signature of the diurnal variation, Sq (Curto et al. 1994b).
It is difficult to separate the variation of the event itself from that which would be seen anyway without this perturbation. Hence, it seems easier to work with the derivatives from the functions as the rate of diurnal variation $\mathrm{Sq}(T=24 \mathrm{~h})$ is much lower than that of the Sfe ( $T$ approx. minutes) - in this way, we do not need to search for the base levels, a laborious task which involves a certain amount of subjectivity (Curto et al. 1994a). The relations ruling the physics of these phenomena are well known and it is possible to model the Standard behaviour of the Sfe (Curto et al. 1994b). However, when we are dealing with a specific event of which we have few data of the variations in the ionosphere, these models do not apply and we have to resort to a simpler approach. Hence, in this work, for the sake of simplicity and taking into account that the contribution of the X-ray and the UV-ray to ionisation is accumulative and does not interact because they act at different levels of the ionosphere, we have supposed that the ordinary differential equation (ODE) governing the process will be of this type:

$$
\frac{\mathrm{d} B(t)}{\mathrm{d} t}=\alpha \frac{\mathrm{d} X(t-a)}{\mathrm{d} t}+\beta \frac{\mathrm{d} U(t-b)}{\mathrm{d} t}+\sigma .
$$

Where $B(t)$ is the earth's magnetic field and $X(t-a)$ and $U(t-b)$ are the functions of the energy flux of X-rays and UV-rays, respectively. They are all dependent on time, $t$. The three (dimensional) parameters $\alpha, \beta$ and $\sigma$ will be determined statistically for each flare using multiple linear fits. For the sake of simplicity, we shall only choose magnetic observations above the same meridian as the vortex of the system of currents, guided by the position of the subsolar point. In this way, the magnetic contribution of the Sfe will be mainly made up of the North component of the magnetic field, X. Moreover, we will choose observatories at a medium distance $(2000-3000 \mathrm{Km})$ from the focus so that their magnetic signatures could be expected to be of the same order of magnitude and only dependent on the intensity of the flare. The term $\sigma$ (the intercept) is a constant to which the variation in the magnetic field caused by other phenomena is attributed, and which is considered to be constant during the flare. A priori, we will choose events which have happened within intervals of time calm enough for any rapid variations with magnetospheric origin (which could distort the calculations) to be considered to be negligible. The parameters $a$ and $b$ are the quantities of time which explain the inertia of the ionosphere to respond to external excitations via ionization radiation which, in our case, synchronize cause and effect.

Next, we apply our model to several Sfe events with different intensities to obtain a representation covering the main range of variation and, thus, to be able to study the behaviour of the parameters in Eq. (1). For this, we chose events where we had all the data (UV-ray, X-ray and magnetism) available and where, specifically, the earth's magnetic field was measured at a point on Earth. The events were selected in order to have samples in each range of energies producing Sfe. So few samples were needed. After this we calculated the numerical derivative of $X(t+a), U(t+a)$ and $B(t)$, and applied a multiple lineal regression with the earth's magnetic field as the dependent variable. In this way, we calculated all the parameters $\alpha \quad(\mathrm{nT} \mathrm{m} / \mathrm{W}), \quad \beta \quad\left(\mathrm{nT} \mathrm{m}^{2} / \mathrm{W}\right), \quad \sigma \quad(\mathrm{nT} / \mathrm{min}$.$) ,$ $a$ (min.) and $b$ (min.) (Table 1).

Inertial parameters have 0-1-2 minute values. As in Le et al. (2011), the $\alpha$ and $\beta$ parameters vary with the intensity of the flare, decreasing as the flare has more power. Regression coefficients, $R^{2}$, are quite high which confirms that our mathematical model is sufficiently accurate to reproduce the 
J.J. Curto et al.: Sfe: waiting for the big one

Table 1. Parameters calculated for seven different solar flares. The last column indicates the acronym of the magnetic observatory used in each event.

\begin{tabular}{|c|c|c|c|c|c|c|c|c|}
\hline Date & Power & $\alpha \times 10^{-4}$ & $\beta \times 10^{10}$ & $\sigma$ & $R^{2}$ & $\bar{a}$ & $b$ & OBS \\
\hline 20 July 2004 & M8.6 & $13.7 \pm 0.67$ & $5.8 \pm 1.5$ & $0.52 \pm 0.24$ & 0.73 & 2 & 1 & ESK \\
\hline 04 June 2007 & M9.0 & $7.5 \pm 1.2$ & $6.60 \pm 2.1$ & $0.10 \pm 0.21$ & 0.84 & 1 & 0 & KAK \\
\hline 08 November 2013 & $\mathrm{X} 1.1$ & $8.7 \pm 1.7$ & $6.4 \pm 2.4$ & $0.22 \pm 0.36$ & 0.81 & 1 & 1 & IRT \\
\hline 24 September 2011 & X1.9 & $6.0 \pm 2.3$ & $5.8 \pm 2.6$ & $0.47 \pm 0.11$ & 0.88 & 2 & 1 & SOD \\
\hline 20 January 2005 & X7.1 & $2.0 \pm 0.6$ & $2.4 \pm 1.1$ & $0.1 \pm 0.4$ & 0.69 & 2 & 0 & ABG \\
\hline 15 April 2001 & X14.4 & $2.5 \pm 0.6$ & $3.5 \pm 2.2$ & $1.3 \pm 0.50$ & 0.89 & 2 & 0 & ESK \\
\hline 2 April 2001 & $\mathrm{X} 20.0$ & $1.7 \pm 0.2$ & $2.6 \pm 1.5$ & $-0.18 \pm 24$ & 0.74 & 2 & 1 & $\mathrm{CBB}$ \\
\hline
\end{tabular}

(a)

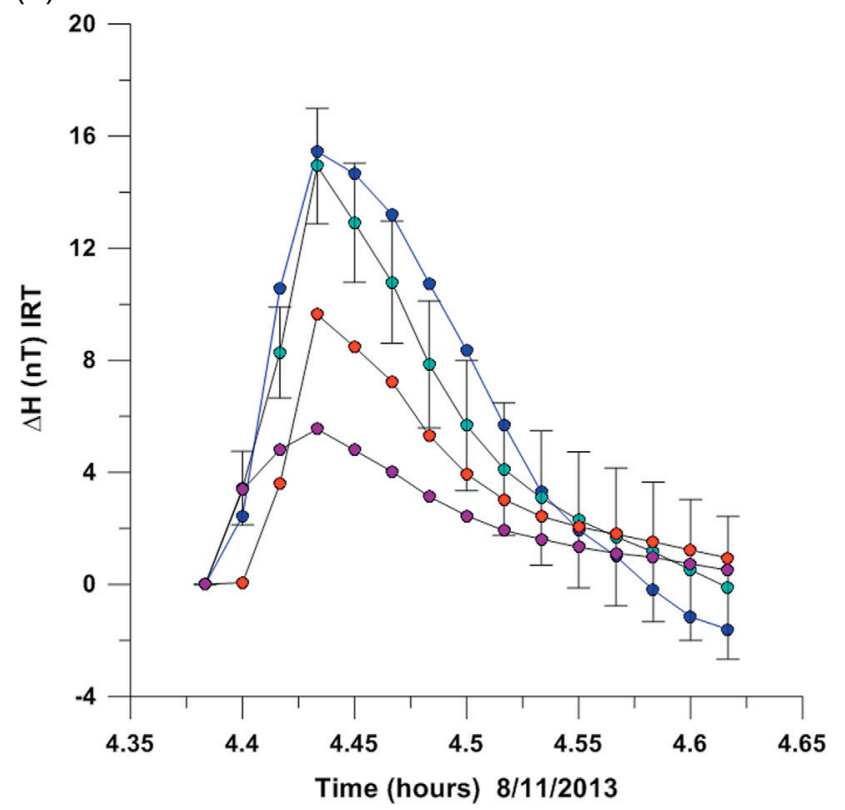

(b)

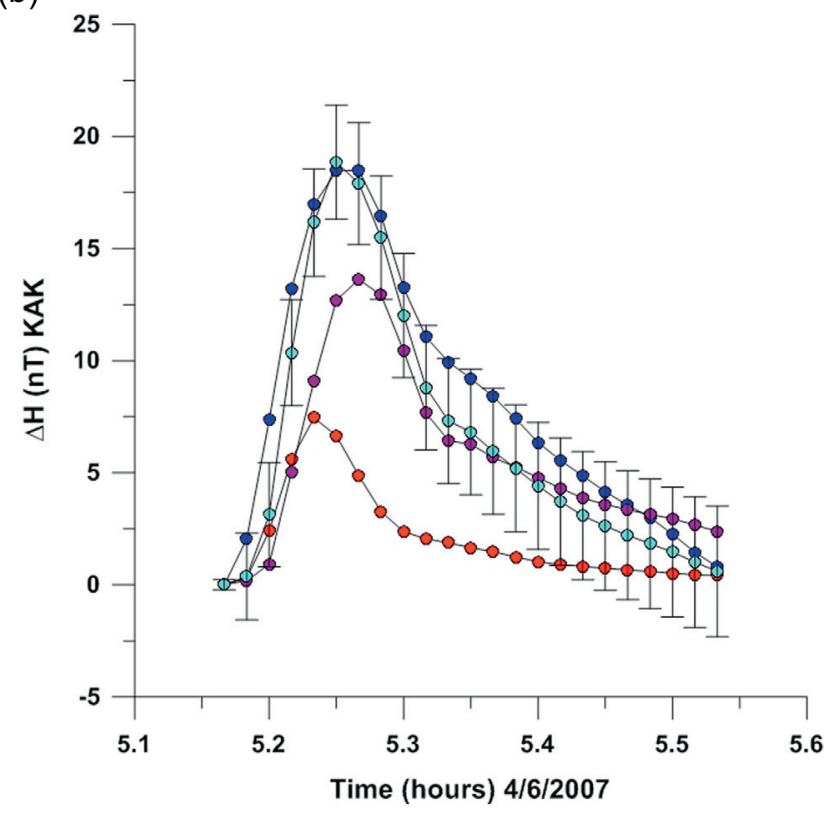

Fig. 1. Comparison between real/experimental magnetic field and modelled/theoretical magnetic field calculated using Eq. (1) for two solar flares: (a) 8 November 2013 (X1.1; Observatory: IRT) and (b) 4 June 2007 (M9.0; Observatory: KAK).

event. However, according to our experience gained in revising many cases, the fitting works well when the event is pure in the sense that only an excess of radiation is present at that moment. However, if the event was preceded by others, then effects of particle precipitation appear and interfere with the Sfe signal at the time of the flare creating magnetic variations that do not follow our simple relationship.

\subsection{Test of validity of the model}

As a test of the procedure we have modelled different events where we know all the data. The comparison between real values and modelled ones for two of these events is shown in Figure 1. The dark blue points correspond to the real magnetic field as measured by the observatory, while the light blue points represent the magnetic field calculated from the differential Eq. (1) using the values of X-ray and UV-ray flux measured by satellite. The red points represent the X-ray flux contribution to the magnetic field, and the purple ones the UV-rays contribution. The calculated values reproduce the measurements very well and we can conclude that the method is valid for making relevant estimations.

As we can see, the contribution of the X-ray component is greater than that of the UV-rays in the first case (Fig. 1a), especially in the first part of the perturbation. In the second case $(8 / 11 / 2013)$ (Fig. 1b) it is the UV-ray component which dominates.

\subsection{Inverse modelling}

As we have stated before, the objective of this work is to be able to estimate the flux of X-rays starting out from the value of magnetic field variations and UV-ray radiation. To do that, we have to use the same Eq. (1) but, this time, the dependent and independent variables interchange their role:

$$
\frac{\mathrm{d} X(t-a)}{\mathrm{d} t}=1 / \alpha \frac{\mathrm{d} B(t)}{\mathrm{d} t}-\frac{\beta}{\alpha} \frac{d U(t-b)}{d t}-\sigma / \alpha .
$$

Using this tool we can carry out the modelling of the case we are especially interested in: the Halloween event. This event has been one of the largest events ever recorded according to most lists of events classified by intensity, such as that of Cliver (2006). However, as previously mentioned, the power of the radiation of this flare has not been accurately measured yet. During the Halloween event, the GOES satellite sensor saturated and it was not possible to measure the maximum value of the flare (NOAA estimated it as X28 in a later re-analysis).

For the Carrington event, the direct use of Eqs. (1) and (2) is not possible because it occurred long before the use of 


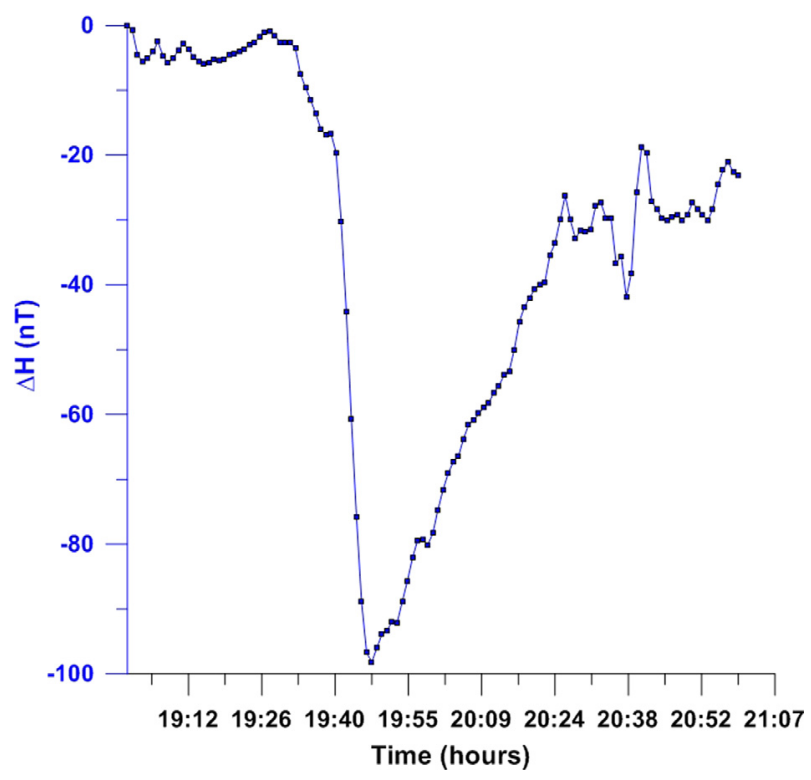

Fig. 2. Sfe corresponding to 4 November 2003 as recorded at Victoria magnetic observatory in Canada. This event reached its maximum at 19:48 and is popularly known as the Halloween Sfe.

satellites and it was, obviously, impossible to take radiation measurements. However, the earth's magnetic field was measured during this event and so the sizes of the Sfe causing the event are known. This is what we will use to estimate the intensity of the flares which caused these perturbations. To solve this particular case, we will use UV and X-ray relationships found by Le et al. (2011) who, after a statistical analysis of a large set of flare samples, established linear regressions of UV and X-rays (with $A$ and $B$ parameters) which correlate quite well when the flare distance to the central solar meridian (central meridian distance, CMD) is taken into consideration.

Finally, we can find the following relationship linking the $\mathrm{X}$-ray and Sfe variations:

$$
\begin{gathered}
\alpha \frac{\mathrm{d} f(t-a)}{\mathrm{d} t}+\beta\left(A+B \frac{\mathrm{d} f(t-a)}{\mathrm{d} t}\right)+\sigma=\frac{\mathrm{d} B(t)}{\mathrm{d} t}, \\
\frac{\mathrm{d} f(t-a)}{\mathrm{d} t}=\frac{\frac{\mathrm{d} B(t)}{\mathrm{d} t}-\sigma-\beta A}{(\alpha+\beta B)} .
\end{gathered}
$$

According to Le et al. (2011), parameters $A$ and $B$ depend on the CMD and the radiation intensity and, thus, they must be calculated taking into account the position the flare had on the Sun.

\section{Results concerning the power of the Halloween and Carrington flares}

Although both events have many characteristics in common, their different data availability forced us to use different strategies to obtain their X-ray flux. To show the way we proceeded more clearly, a diagram comparing both procedures follows:

Halloween event

(a1) With data during the time of no-saturation $\left(X_{\text {Halloween }}+U_{\text {Halloween }}+B_{\text {Halloween }}\right)+$ equ. 1 - $>\alpha, \beta$ and $\sigma$.

(b1) Then, with $\alpha, \beta$ and $\sigma$ from (a1) $+\left(U_{\text {Halloween }}+\right.$ $\left.B_{\text {Halloween }}\right)+$ equ. 2 -> $X_{\text {Halloween }}$ for the whole period.

Carrington event:

(a2) $X_{\text {Carrington1 }}=X_{\text {Halloween }}+$ equ. 5 -> $\alpha 1, \beta 1$ and $\sigma 1$.

(b2) With $\alpha 1, \beta 1$ and $\sigma 1$ from (a2) + equ. $4->X_{\text {Carrington2. }}$

(c2) $X_{\text {Carrington2 }}$ from (b2) + equ. $5->\alpha 2, \beta 2$ and $\sigma 2$.

(b2) With $\alpha 2, \beta 2$ and $\sigma 2$ from (c2) + equ. $4->X_{\text {Carrington3 }}$.

\subsection{The Halloween event}

At this point it is worth remembering that for this event, the X-ray flux sensor produced data during the event except for some minutes of the maxima (between 19:43 and 19:56). The calculation of the Halloween event will be done in two stages: (a) first, with the available information $(B, X$ and $U$ ) during the time of no-saturation we will calculate the $\alpha, \beta$ and $\sigma$ parameters with the direct relationship 1; (b) later, using the geomagnetic field variation and UV flux data information of the whole event, the parameters calculated in the first stage and the inverse relationship 2 we will find the evolution of the radiation. To calculate the Halloween flare we used magnetic data from the Canadian observatory, Victoria (Fig. 2), which was at the north of the vortex of the current system at that moment.

If we do a multiple linear fit with the data we have (throughout the event except for 14 points), we obtain the results expressed in Table 2.

With the parameters calculated, we can now model the whole event (Fig. 3).

The maximum point was at 19:46 and reached an intensity of X46.3 \pm 1.8

On the other hand, taking into account results from Tables 1 and 3 , a relationship between power and the values of the respective parameters can be seen (Fig. 4).

Both parameters fit well with an exponential function:

$$
Y=Y_{0}+A \times e^{-P / t},
$$

the values of the constants for each parameter are given in Table 3. $Y$ is the value of the parameter and $P$ the intensity of the flare adjusting the constants $Y_{0}, A$ and $t$.

Once again we have a high correlation according to the coefficient values of regression $R^{2}$.

\subsection{The Carrington event}

To model the Carrington event, we only have the geomagnetic data available, so we cannot calculate the parameters directly with Eq. (1). Hence, we have to use a slightly different strategy.

The data of the magnetic variation (Fig. 5) were obtained by digitalizing the records of the magnetic observatory at

Table 2. Parameters for the Halloween event.

\begin{tabular}{lccccccc}
\hline \hline Date & Power & $\alpha \times 10^{-4}$ & $\beta \times 10^{10}$ & $\sigma$ & $R^{2}$ & $a$ & $b$ \\
\hline 4 November 2003 & $\mathrm{X} 46.3$ & $1.67 \pm 0.12$ & $2.3 \pm 1.2$ & $0.86 \pm 0.35$ & 0.95 & 2 & 1 \\
\hline
\end{tabular}


J.J. Curto et al.: Sfe: waiting for the big one

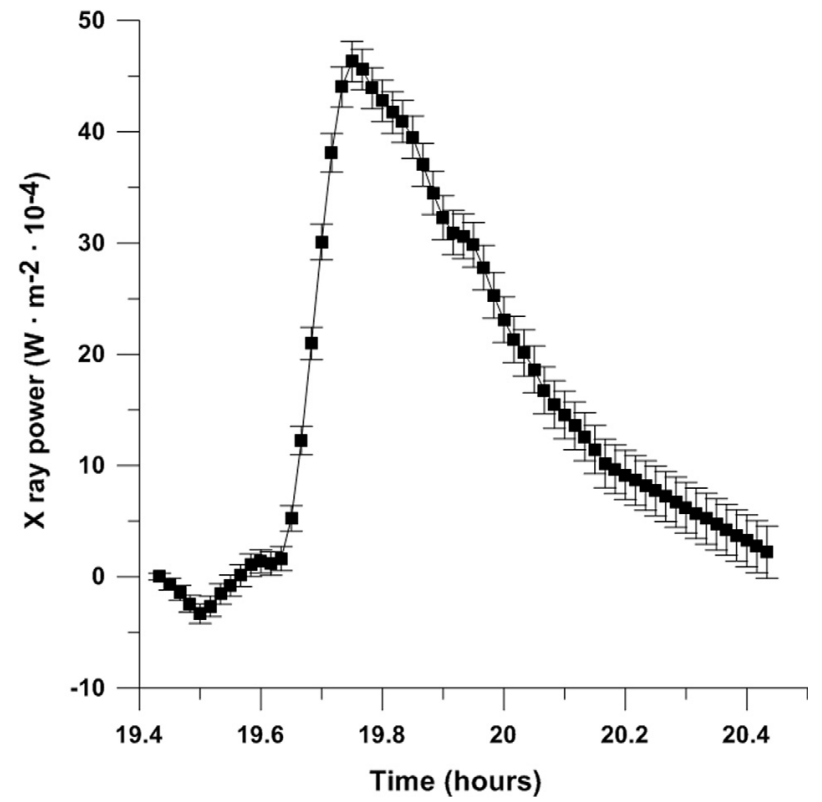

Fig. 3. Complete temporal evolution of X-ray flux for the Halloween event on 4 November 2003 according to our mathematical method.

Table 3. Values of the different constants of the statistical fit of parameters $\alpha$ and $\beta$.

\begin{tabular}{lcccc}
\hline \hline$Y$ & $Y_{0}$ & $A$ & $t$ & $R^{2}$ \\
\hline$\alpha \times 10^{-4}$ & $1.82 \pm 0.45$ & $11.4 \pm 2.5$ & $1.91 \pm 0.77$ & 0.94 \\
$\beta \times 10^{10}$ & $2.7 \pm 0.30$ & $5.6 \pm 0.8$ & $3.0 \pm 1.2$ & 0.94 \\
\hline
\end{tabular}

Kew (UK) for that day using our measuring technique (Curto et al. 1996).

The Carrington event is very similar to the Halloween one as regards the magnetic variation amplitude, thus we can

(a)

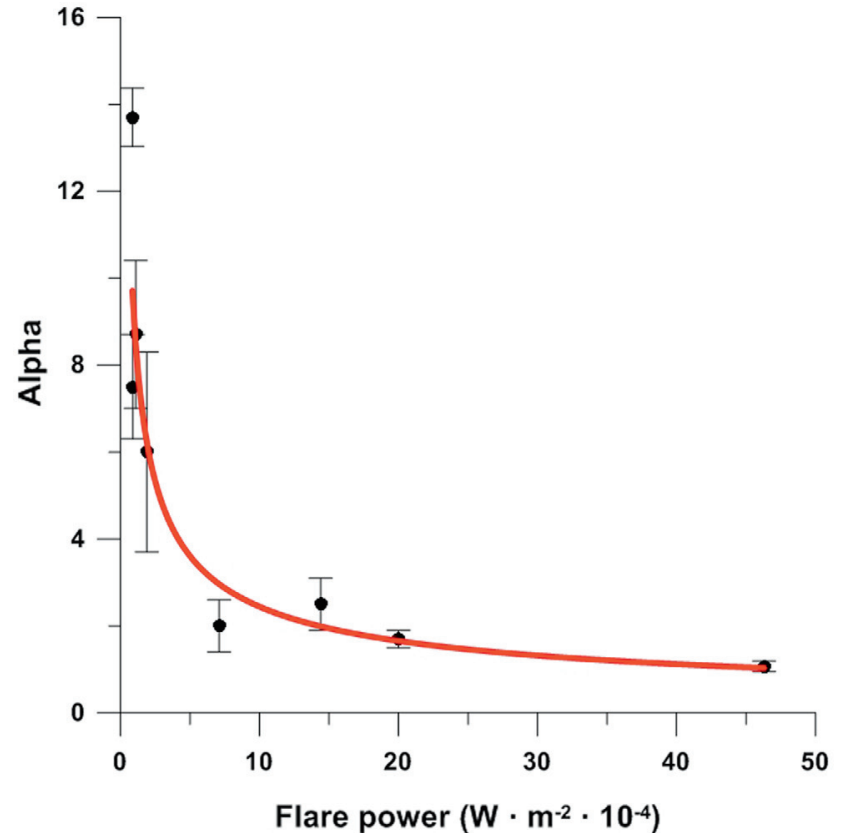

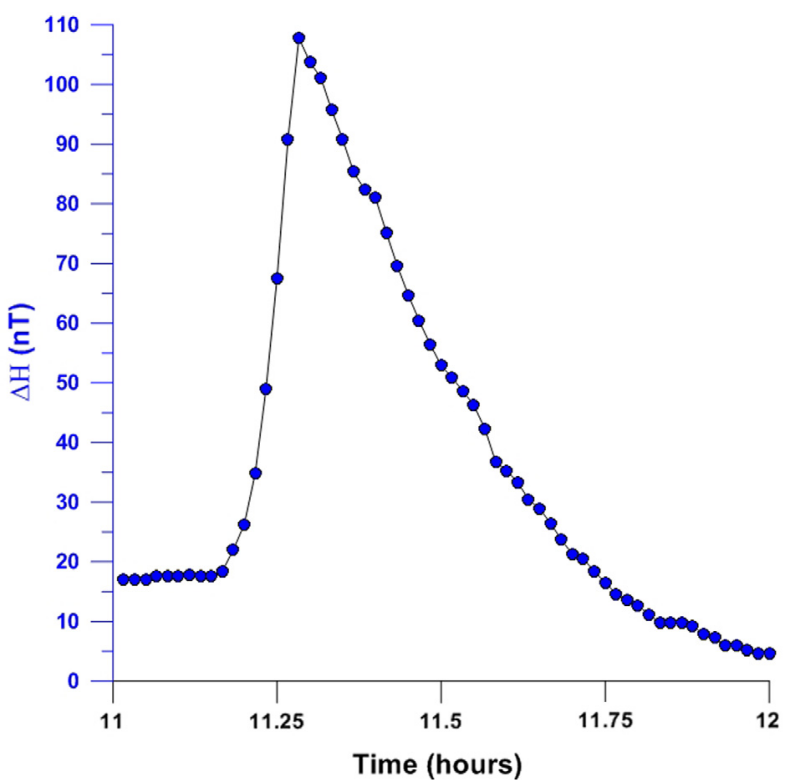

Fig. 5. Sfe corresponding to 1 September 1858 as recorded at the magnetic observatory in Kew (UK). This event had a maximum peak at 11:17 and is popularly known as the Carrington Sfe.

suspect that the delivered energy in both cases was similar, so we will use the same value as an initial trial. In fact, the estimation of the Carrington event power was done in an iterative process. First we applied to Eq. (4) the value of the parameters derived from Eq. (5), taking as the initial exploratory value of the Carrington power a value equal to that of the Halloween event. Thus we obtained a second estimated value of the Carrington power. With this second value we initiated another iteration, giving a third value. As this third result coincided with the second one, we stopped the iteration and took this to be the definitive one (Fig. 6).

The maximum happened at 11:15, reaching an intensity of $\mathrm{X} 45.7 \pm 2.2$.

(b)

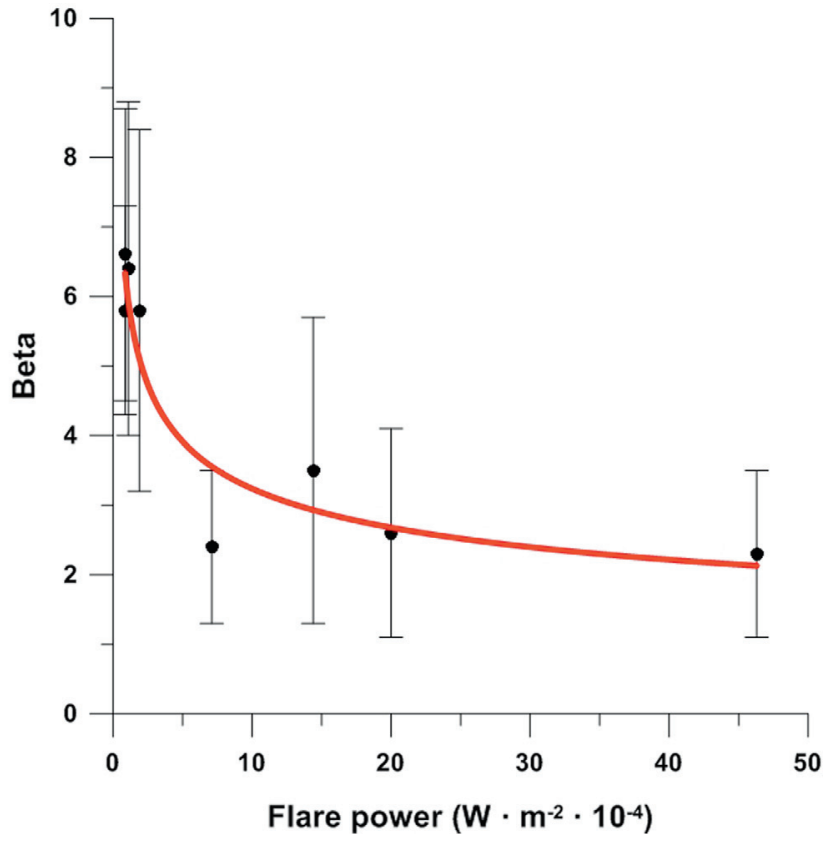

Fig. 4. Values of the parameters $\alpha$ (a) and $\beta$ (b) as a function of the power of the flare. The red line is a fit following a power law. 


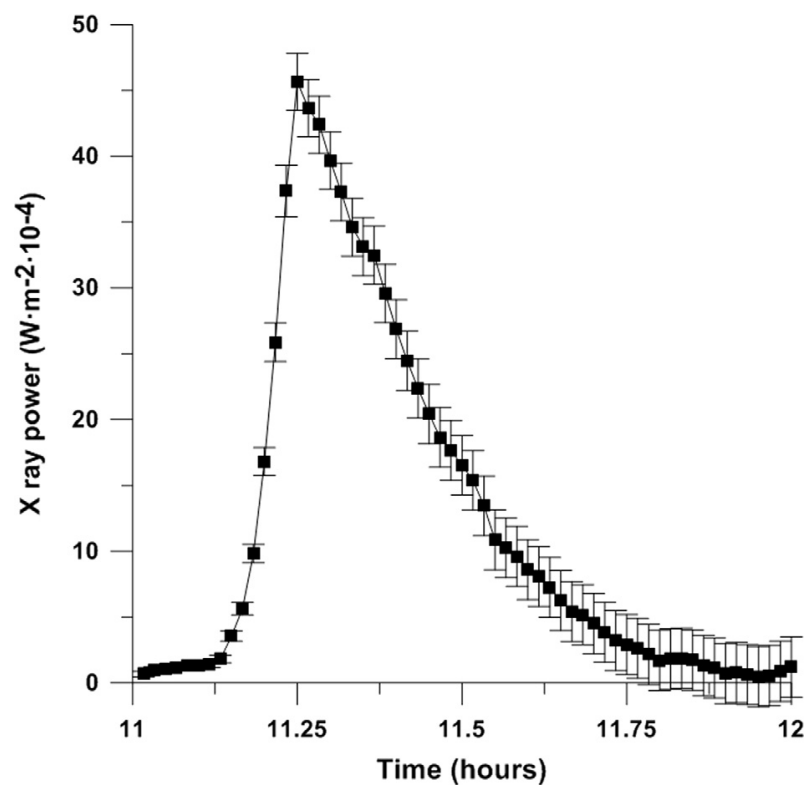

Fig. 6. X-ray flux of Carrington event on 1 September 1858 modelled using our mathematical method, with a maximum of $\mathrm{X} 45.7 \pm 2.2$ at $11: 15$.

Following our mathematical model, we obtain the result that the Halloween event was a X46.3 \pm 1.8 flare class and the Carrington event was a X $45.7 \pm 2.2$ flare class. To sum up, at the maximum point, according to our calculations both the Carrington and the Halloween events were higher than $\mathrm{X} 45$, doubling the intensities of the next most powerful flares below them on the list of greatest flares ever recorded, as well as being way higher than any of the other recorded flares. This confirms the exceptionality of these events.

\section{Return period: when}

As humankind are users of a multitude of ever-morecomplex technological systems, which are also increasing in

(a)

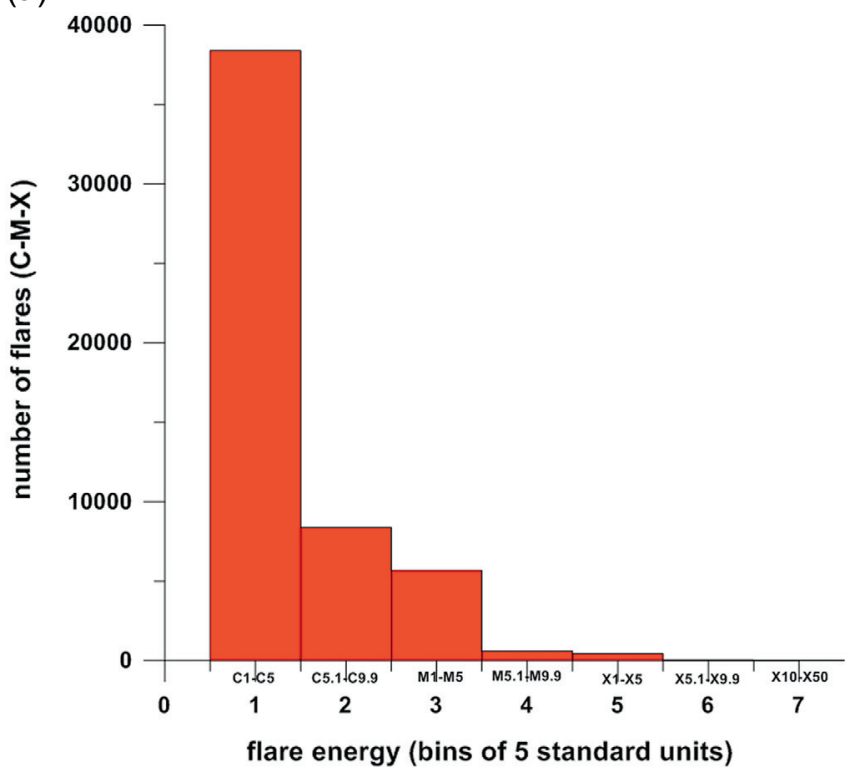

vulnerability, we are interested in knowing when we can expect another event so large that it could cause significant perturbations in telecommunication networks, electricity supplies or damage to satellites which offer essential services for a society as technology-dependent as ours. We are particularly interested in knowing when a Carrington-type major event will occur again.

\section{Calculation}

To calculate the return period of a Carrington-like flare we used NOAA flare lists which cover the whole period: 1 October 1975 to 21 May 2015, separating the flares into bins of 5 flare classification units starting with $\mathrm{C} 1$, i.e. C1-C5, C5.1-C9.9, M1-M5, M5-M9.9, X1-X5, X5-X9.9,... (Fig. 7a). We used the original data except for the case of the Halloween event for which we used our calculations instead of the NOAA data. In general, the number of flares follows a power law with respect to their energy. Like a fractal structure, this happens in each sub-band we take.

Due to a large "weight" of the $\mathrm{C}$ and $\mathrm{M}$ flares compared to the $\mathrm{X}$ flares (Fig. 7a), any attempt to adjust a function will underestimate the X-ray population, resulting in a nonrealistic return period calculation for X-rays. Thus, we only consider X-type flares (Fig. 7b) and, therefore, the functions we use to adjust data are more representative for this range. This allowed us to find reliable results that match with our expectations.

To calculate the return periods for the X-type flares, we used the Generalized Extreme Value distribution (GEV) (also known as the Fisher-Tippett distribution) because of its versatility. GEV is perfect for our aims because it is very appropriate to model the smallest or largest value among a large set of observations with values randomly distributed, as in our case. GEV combines three simpler distributions - the Gumbel (Gumbel 1954), Fréchet (Fréchet 1927) and Weibull (Weibull 1951) functions - into a single form allowing a continuous range of possible shapes that includes all three of the simpler distributions. Thus, the generalized extreme value distribution

(b)

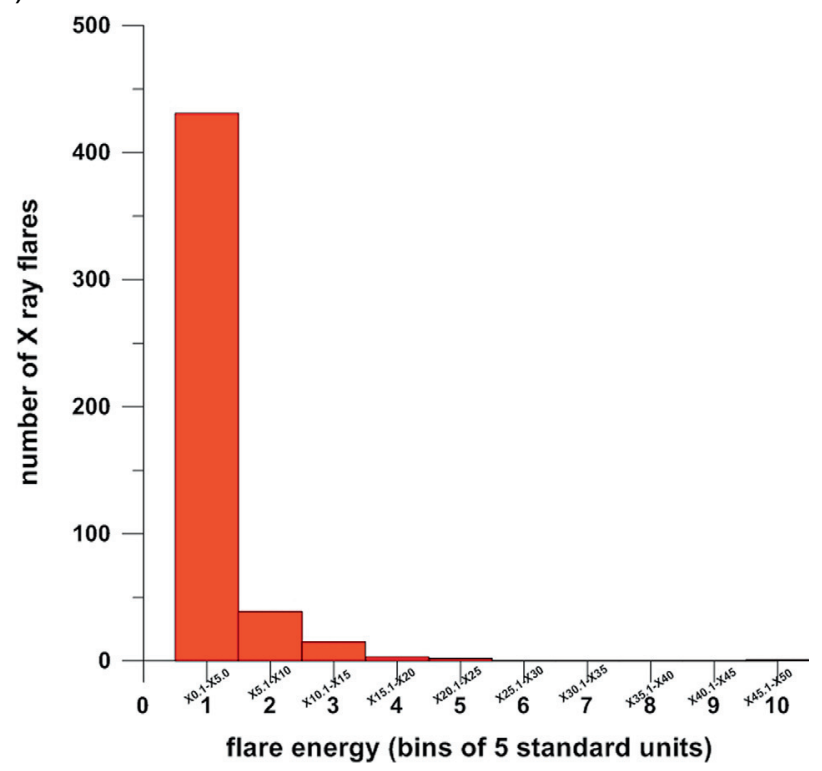

Fig. 7. (a) Number of flares for each bin. Each bin contains 5 standard units. (b) Number of X-class flares for each bin. Each bin contains 5 standard units. 
(a)

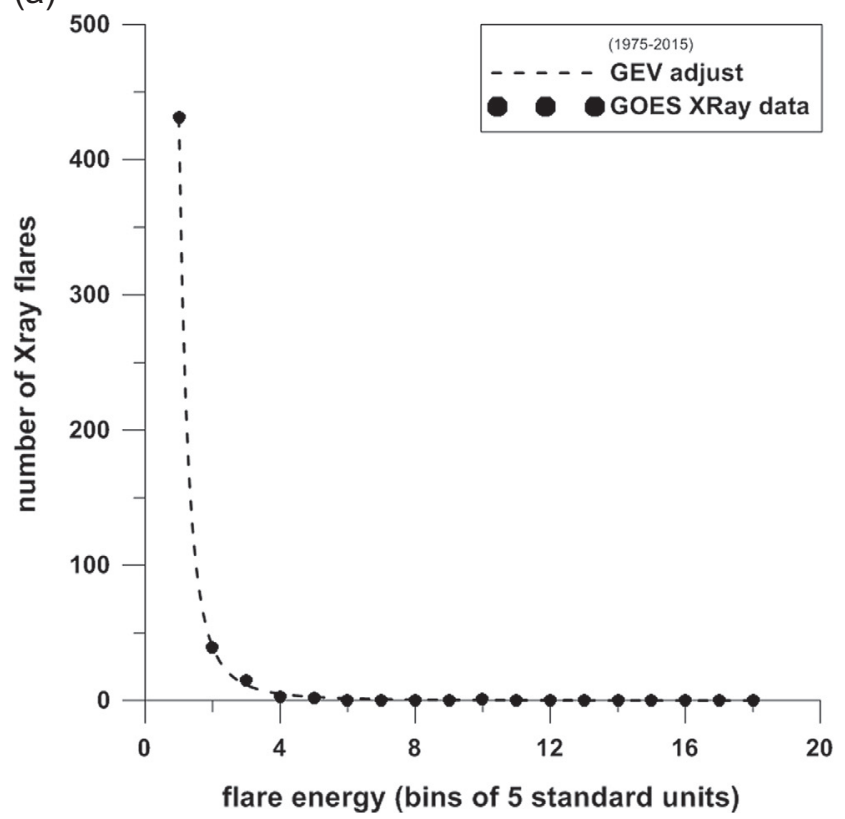

(b)

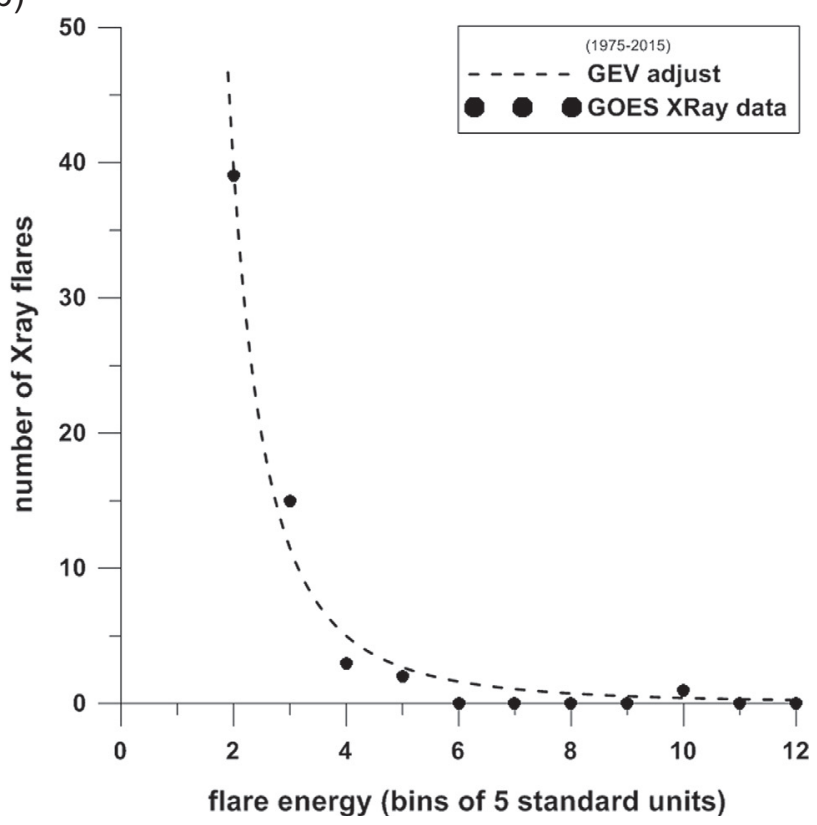

Fig. 8. (a) Number of observed X-ray flares with GEV regression. (b) Same representation as in Figure 8a, but with a zoom in the energy range of the Carrington-like flare.

allows us to "let the data decide" which distribution is appropriate. Although we tried other distributions, only the GEV distribution adjusted the flare distribution properly.

\subsection{GEV fit and results}

The adjusted GEV distribution (Ibáñez Rosales 2011) can be written as:

$$
f(x ; \mu, \sigma, \xi)=\frac{k}{\sigma} p^{(-1 / \xi)-1} \exp \left\{-p^{-1 / \xi}\right\},
$$

where $p=1+\xi\left(\frac{x-\mu}{\sigma}\right)$.

And $k$ is the total number of flares considered. In our case, the number of X-ray (class $X$ ) flares reported in the NOAA database is $k=491$. We must multiply the function by this factor because the original GEV function (without the 491 factor) is normalized to 1 .

Using this method to fit the GEV distribution to the data, the coefficient values are: $\sigma=0.198 \pm 0.017, \mu=0.799 \pm$ 0.008 and $\xi=0.66 \pm 0.10$. Figure 8 a shows the adjusted curve. The zoom in the Carrington-like energy range (Fig. $8 b$ ) reveals how specific the occurrence of such an event is.

The coefficient of determination is $R^{2}=0.99987$. Hence, we can see we had a very precise adjustment.

With the parameters obtained, the return value of a flare with an intensity compressed between a 5-unit bin can be calculated as:

$$
T=\frac{\tau}{f(x ; \sigma, \mu, \xi)}
$$

Where $\tau$ is the period of time since the beginning of the data until the end and $x$ belongs to the bin studied. In our case $\tau=40$ years. For a flare between X45 and X50, $x=10$. This expression gives us a return period of $T=90 \pm 60$ years.

\section{Conclusions}

In this work we have calculated the X-ray intensity for the Carrington and Halloween events. We then estimated the return period of a Carrington-like event.

The fact that there has always been controversy regarding the maximum values reached by the two largest events ever measured encouraged us to carry out a new estimation based on corresponding magnetic data on the Earth (Sfe). We first looked for a relationship between the intensity of the radiation incident at earth level (cause) and the amplitude of the magnetic variation produced (consequence). Using this relationship we used inverse modelling to estimate the radiation values at the maximum moment. These were similar for both cases and were above X45. Despite this, it has to be remembered that there was a significant difference between these two flares: Carrington reported seeing, with the naked eye, two intensely white and bright patches of light on the image of the Sun's disk projected onto a plate of glass. The Carrington flare emitted such a large quantity of radiation in the visible range that it became the first flare ever reported and the first to be linked to a terrestrial response (Cliver 2006).

Afterwards we calculated the Sfe occurrence statistics for the period where we have data from satellites (1973-2015) and we looked for a GEV-type function which, when adjusted to the distribution of the flare happenings in the $X$ band, allowed us to calculate the return periods for extreme events such as the Carrington event.

As mentioned previously, we obtained a return period $T_{\mathrm{GEV}}=90 \pm 60$ years for a Carrington event to happen and a Carrington-like flare (X45) was seen in 2003, 144 years after the original Carrington flare (1859).

This study allowed us to estimate the return period of a Carrington-like flare as being 90 years. Having said that, though, we have to bear in mind that our data set is very 
reduced because radiation data were only available for the last 40 years and the fact that, before this period, only one event, that of the Carrington flare in 1859, had been observed. To obtain more reliable results, a study with more years of data should be carried out. The ideal case would be with hundreds of years of data, - in which case the sample would be more representative (e.g. more great flares ( $>$ X25) would have been registered). However, for now, with this limited amount of data, the return period of $T=90 \pm 60$ years is the best estimate of the real return period that we were able to obtain.

Recent papers looking at several other parameters, including observed CME speeds and the strength of the equatorial current system in the Earth's magnetosphere, concluded that the risk of an extreme space weather episode could be as high as $12 \%$ in a decade (Riley 2012). From this result, the UK Royal Academy of Engineering issued a report on extreme space weather in 2013. They deduced a return period or recurrence interval of 79 years for a superstorm. The usual 95\% confidence interval implies we might only have to wait two years for a superstorm, or we might have to wait 300 years. This is a consequence of the nature of randomness. On the other hand, Thomson et al. (2011) quantified the probability of having extreme behaviour in geomagnetic activity as ranging from 100 to 200 years. Thus, different approaches converge in the conclusion that extreme events are very rare and we do not expect one to happen in the near future.

We can conclude that, according to our estimate, humanity will probably have to wait another century to see a big flare located in the Sun's visible hemisphere with the capacity to produce impacts on Earth via a new Carrington-like Sfe.

Acknowledgements. This research has been partially supported by Spanish government project CTM2014-52182-C3-1-P of MINECO. The authors also wish to thank the NOAA for the satellite system which collects valuable information regarding solar radiation and for publishing these data. We acknowledge the CELIAS/ SEM experiment on the Solar Heliospheric Observatory (SOHO) spacecraft (SOHO is a joint European Space Agency, United States National Aeronautics and Space Administration mission). We also thank INTERMAGNET and all the collaborating observatories which provide high-quality magnetic data. Finally, we thank the International Service of Rapid Magnetic Variations and their collaborating observatories for providing lists of Sfe events, essential for a study such as ours.

The editor thanks two anonymous referees for their assistance in evaluating this paper.

\section{References}

Brodrick, D., S. Tingay, and M. Wieringa. X-ray magnitude of the 4 November 2003 solar flare inferred from the ionospheric attenuation of the galactic radio background. J. Geophys. Res., 110, A09S36, 2005, DOI: 10.1029/2004JA010960.

Cannon, P.S. Extreme Space Weather - a report published by the UK Royal Academy of Engineering. Space Weather, 11, 138-139, 2013, DOI: 10.1002/swe.20032.

Carrington, R.C. Description of a singular appearance seen in the Sun on September 1, 1859. Mon. Not. Roy. Astron. Soc., 20, 13-14, 1860.

Clarke, E., C. Rodger, M. Clilverd, T. Humphries, O. Baillie, and A. Thompson. An estimation of the Carrington flare magnitude from solar flare effects (sfe) in the geomagnetic records 2010, Royal Astron. Soc. National Astron. Meeting, University of Glasgow, UK, 2010.
Cliver, E.W. The 1859 space weather event: then and now. Adv. Space Res., 38, 119-129, 2006.

Cliver, E.W., and W.F. Dietrich. The 1859 space weather event revisited: limits of extreme activity. J. Space Weather Space Clim., 3, A31, 2013, DOI: 10.1051/swsc/2013053.

Cliver, E.W., and L. Svalgaard. The 1859 solar-terrestrial disturbance and the current limits of extreme space weather activity. Sol. Phys., 224, 407-422, 2004.

Curto, J.J., C. Amory-Mazaudier, J.O. Cardús, J.M. Torta, and M. Menvielle. Solar flare effects at Ebre: regular and reversed solar flare effects, statistical analysis (1953 to 1985), a global case study and a model of elliptical ionospheric currents. J. Geophys. Res., 99 (A3), 3945-3954, 1994a.

Curto, J.J., C. Amory-Mazaudier, J.O. Cardús, J.M. Torta, and M. Menvielle. Solar flare effects at Ebre: unidimensional physical, integrated model. J. Geophys. Res., 99 (A12), 23289-23296, 1994b.

Curto, J.J., E. Sanclement, and J.M. Torta. Automatic measurement of magnetic records on photographic paper. Comput. Geosci., 22 (4), 359-368, 1996.

Curto, J.J., L.F. Alberca, and J. Castell. Dynamic aspects of the Solar flare effects and their impact in the detection procedures. J. Ind. Geophys. Union, 2, 99-104, 2016.

Fréchet, M. Sur la loi de probabilité de l'écartmaximum. Ann. Soc. Polon. Math., 6, 93, 1927.

Gopalswamy, N., L. Barbieri, E.W. Cliver, G. Lu, S.P. Plunkett, and R.M. Skoug. Introduction to violent Sun-Earth connection events of October-November 2003. J. Gepohys. Res., 110, A09S15, 2005, DOI: 10.1029/2004JA010958.

Gumbel, E.J. Statistical theory of extreme values and some practical applications, Applied Mathematics Series 33, 1st ed., U.S. Department of Commerce, National Bureau of Standards, 1954.

Hodgson, R. On a curious appearance seen in the Sun. Mon. Not. Roy. Astron. Soc., 20, 15, 1860.

Ibáñez Rosales, A. Análisis estadístico de valores extremos y aplicaciones, Report of Universidad de Granada, 2011.

Kiplinger, A.L., and H.A. Garcia. Soft X-ray parameters of the great flares of active region 486. Bull. Am. Astron. Soc., 36, 739, 2004.

Lanzerotti, L.J. Geomagnetic influences on man-made systems. J. Atmos. Terr. Phys., 41, 787-796, 1979.

Lanzerotti, L.J. Geomagnetic induction effects in ground-based systems. Space Sci. Rev., 34, 347-356, 1983.

Le, H., L. Liu, H. He, and W. Wan. Statistical analysis of solar EUV and X-ray flux enhancements induced by solar flares and its implication to upper atmosphere. J. Geophys. Res., 116 (A11), A11301, 2011, DOI: 10.1029/2011JA016704.

NOAA. Space environment center preliminary report and forecast of solar geophysical data, Rep. 1471, Silver Spring, Maryland, 2003.

Riley, P. On the probability of occurrence of extreme space weather events. Space Weather, 10, S02012, 2012, DOI: $10.1029 / 2011$ SW000734.

Schulte in den Bäumen, H., D. Moran, M. Lenzen, I. Cairns, and A. Steenge. How severe space weather can disrupt global supply chains. Nat. Hazards Earth Syst. Sci., 14, 2749-2759, 2014.

Thomson, A.W.P., E.B. Dawson, and S.J. Reay. Quantifying extreme behavior in geomagnetic activity. Space Weather, 9, S10001, 2011, DOI: 10.1029/2011SW000696.

Thomson, N.R., C.J. Rodger, and R.L. Dowden. Ionosphere gives size of greatest solar flare. Geophys. Res. Lett., 31, L06803, 2004, DOI: 10.1029/2003GL019345.

Thomson, N.R., C.J. Rodger, and M.A. Clilverd. Large solar flares and their ionospheric D-region enhancements. J. Geophys. Res., 110, A06306, 2005, DOI: 10.1029/2005JA011008.

Tranquille, C., K. Hurley, and H.S. Hudson. The Ulysses catalog of solar hard X-ray flares. Sol. Phys., 258, 141-166, 2009.

Weibull, W. A statistical distribution function of wide applicability. J. Appl. Mech.-Trans. ASME, 18 (3), 293-297, 1951.

Cite this article as: Curto JJ, Castell J \& Moral FD. Sfe: waiting for the big one. J. Space Weather Space Clim., 6, A23, 2016, DOI: $10.1051 /$ swsc $/ 2016018$. 